\title{
Carcinoma mixto adenocarcinoma y neuroendocrino periampular en un paciente con neurofibromatosis tipo 1. Caso clínico
}

'Departamento de Cirugía Digestiva. División de Cirugía. ${ }^{2}$ Departamento de Anatomía Patológica.

Facultad de Medicina. Hospital Clínico Pontificia Universidad Católica de Chile. Santiago de Chile.

Recibido el 27 de agosto de 2010, aceptado el 2 de diciembre de 2010.

Correspondencia a: Dr. Nicolás Jarufe C. Departamento de Cirugía Digestiva, Facultad de Medicina, Pontificia

Universidad Católica de Chile. Marcoleta 367, Casilla 114-D, Santiago. Fono: 3543870.

Fax: 6329620.

E-mail: njarufe@med.puc.cl
MÓNICA MARTÍNEZ M. ${ }^{1}$, ENRIQUE NORERO M. ${ }^{1}$, FÉLIX GABRIEL LEZCANO G. ${ }^{2}$, SERGIO GONZÁLEZ B. ${ }^{2}$, NICOLÁS JARUFE C. ${ }^{1}$

\section{Combined periampullary adenocarcinoma and neuroendocrine tumor in type 1 neurofibromatosis. Report of one case}

Neurofibromatosis is a hereditary autosomal-dominant disease, with high rates of de novo mutations and carries a high risk of neoplasms. It affects both sexes and all races and ethnic groups. It is characterized by multiple cutaneous lesions and tumors, both benign and malignant, especially in the nervous system. We report a 52 years old woman with a type 1 neurofibromatosis, presenting with fever, jaundice and weight loss. On physical examination, the patient was jaundiced and had "café au lait" spots in the skin. A magnetic resonance imaging showed bile duct dilation and a possible ampullar carcinoma. The patient was operated, during the exploration she presented a periampullary tumor and multiple small nodular lesions in the stomach, the tumor was resected with a pancreaticoduodenectomy and the nodular gastric lesions were biopsied. The pathological study revealed a combined adenocarcinoma and neuroendocrine duodenal tumor. The study of the stomach lesions revealed a gastrointestinal stromal tumor. Four months after surgery, the patient is in good conditions.

(Rev Med Chile 2011; 139: 84-88).

Key words: Gastrointestinal stromal tumors; Neurofibromatoses; Pancreaticoduodenectomy.
U no de cada 2.500-3.500 nacidos vivos presenta la mutación que produce la neurofibromatosis tipo 1 (NF1). El 50\% de estas mutaciones son esporádicas. La mutación se encuentra en un gen supresor en el cromosoma $17(17 \mathrm{q} 11.2)$. Esta se asocia a una perdida en la función de una proteína supresora tumoral, lo que causaría el desarrollo de tumores benignos y malignos $^{1}$. En un $50 \%$ de los casos existen nuevas mutaciones. Se han reportado más de 300 mutaciones diferentes en $\mathrm{NF}^{2}$.

La NF1 se caracteriza por presentar múltiples neurofibromas cutáneos, manchas café con leche, pecas axilares o inguinales y nódulos de lisch. Menos común pero potencialmente más serios son las lesiones óseas, vasculopatías, gliomas ópticos y de otros lugares del sistema nervioso central ${ }^{3}$, además de lesiones gastrointestinales como tumores periampulares, en los cuales existe una asociación clara con NF1, pero con pocos casos descritos en la literatura ${ }^{2}$.

A continuación presentamos el caso de una mujer con NF1 que desarrolló un tumor periampular mixto, adenocarcinoma y tumor neuroendocrino de la segunda porción de duodeno. 


\section{Caso clínico}

Mujer de 52 años de edad, con antecedentes de NF1. Refiere cuadro de 2 meses de evolución de fiebre intermitente, asociado a baja de peso de 5 $\mathrm{kg}$. Durante la última semana previa a su ingreso se agrega ictericia y coluria. Se hospitalizó para estudio. En el examen físico destacaba una leve ictericia y múltiples manchas café con leche en la piel (Figura 1). Se realizaron exámenes que confirmaron ictericia obstructiva, con bilirrubina total de $7 \mathrm{mg} / \mathrm{dl}$. Se estudió con resonancia nuclear magnética de abdomen y pelvis, que mostró dilatación de vía biliar de $25 \mathrm{~mm}$ y del conducto de Wirsung de $7 \mathrm{~mm}$, con una lesión altamente sospechosa de neoplasia de ampolla (Figuras 2-3). La endoscopia digestiva alta confirmó la lesión periampular, se tomó una biopsia de la lesión que mostró enteritis moderada con ectasia vascular leve sin elementos de especificidad. Se realizó estudio de diseminación que resulta negativo. A pesar de que la biopsia fue negativa, se decidió cirugía dado la alta sospecha clínica.

La paciente fue sometida a una laparotomía, se realizó maniobra de Kocher y palpación de la cabeza del páncreas. Se encontró una masa de 2-3 $\mathrm{cm}$ localizada en la región periampular. No había diseminación peritoneal ni hepática. En estómago se encontraron múltiples lesiones planas nodula-

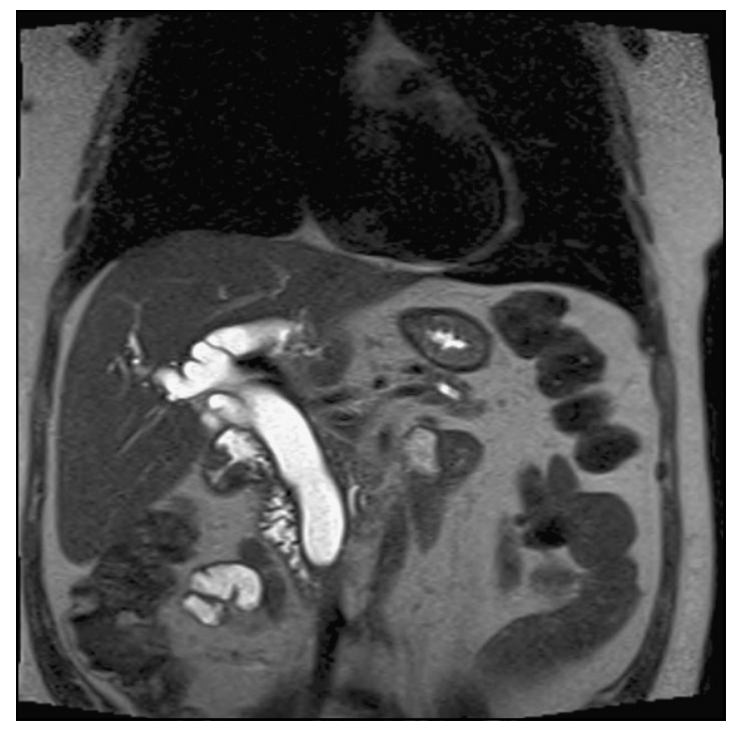

Figura 2. Resonancia nuclear magnética que demuestra dilatación de la vía biliar. res, en cara anterior y posterior, de $2-10 \mathrm{~mm}$, se mandó muestra para biopsia contemporánea que fue informada como tumor fusocelular (Figura 4). Se realizó una pacreatoduodenectomía sin preservación pilórica y posterior reconstrucción

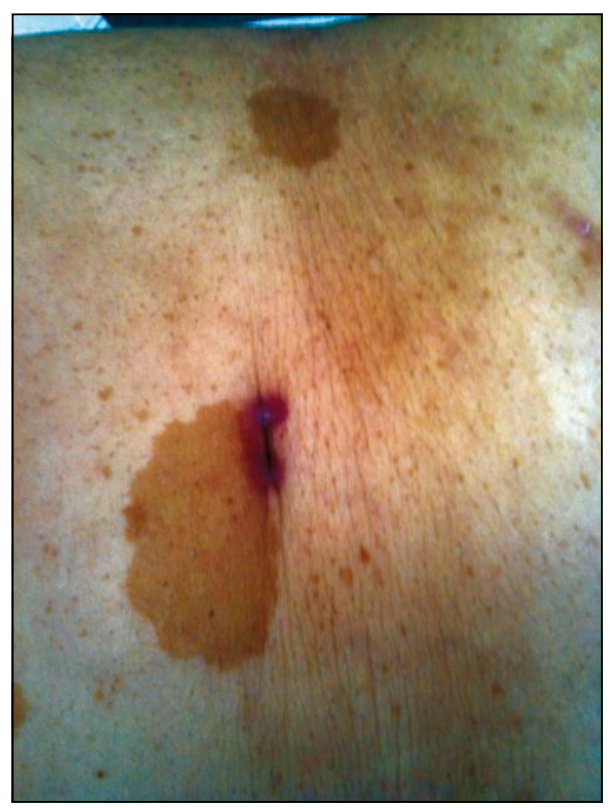

Figura 1. Manchas café con leche en el examen físico de la paciente.

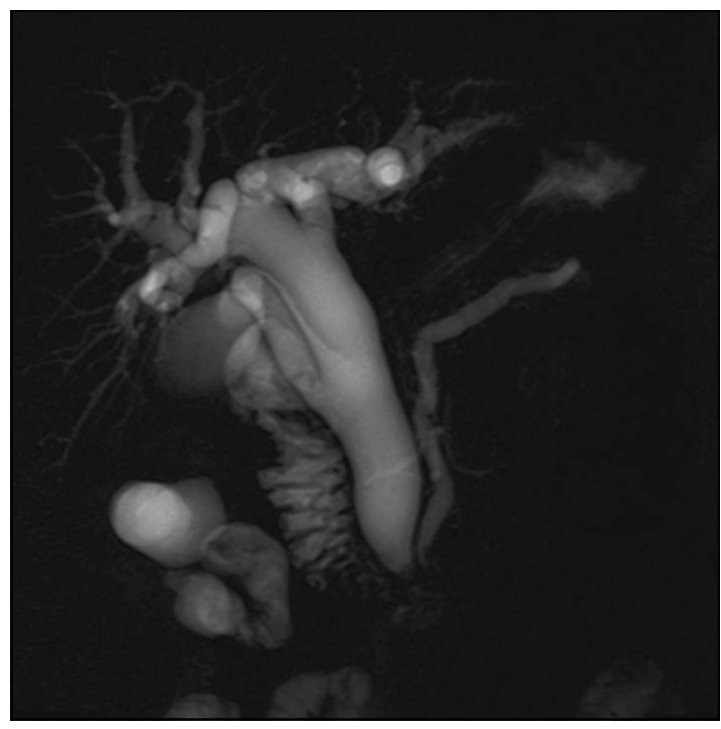

Figura 3. Colangiografía por resonancia nuclear magnética con dilatación de vía biliar y del conducto de wirsung. 


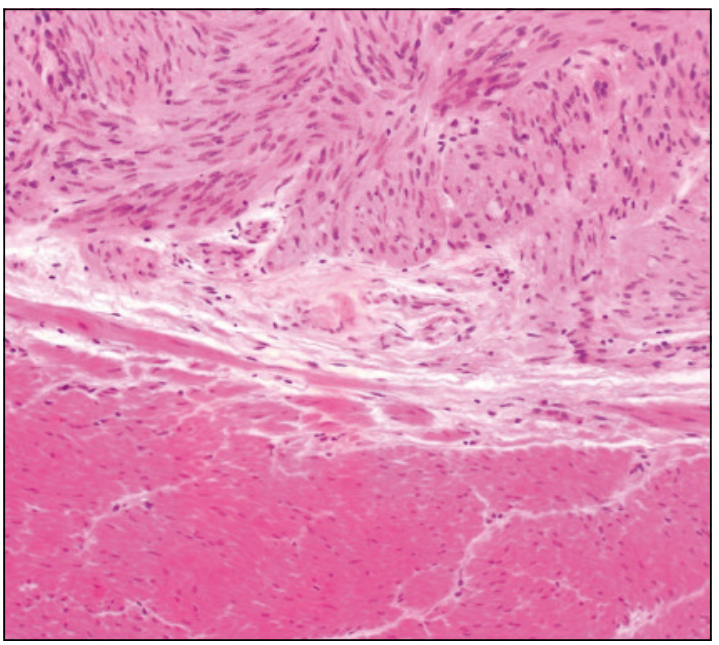

Figura 4. Corte histológico de lesión gástrica informado como tumor fusocelular. Hematoxilina-Eosina. $100 \mathrm{X}$.

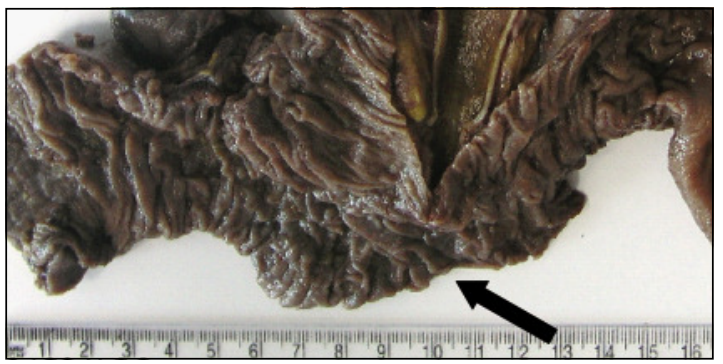

Figura 5. Pieza operatoria de pancreatoduodenectomía. Abierto el duodeno y la vía biliar distal. Tumor duodenal (flecha). sin incidentes. En la evolución postoperatoria la paciente presentó una fístula pancreática de bajo débito que se manejó en forma conservadora por el drenaje y fue dada de alta el día 14 postoperatorio.

La biopsia informaba que en la región periampular, en el duodeno, existía un carcinoma neuroendocrino bien diferenciado combinado con adenocarcinoma tubular bien diferenciado que comprometía la submucosa y la muscular propia del duodeno, de 1 x 1 x 0,8 cm, que alcanzaba la desembocadura distal del colédoco intrapancreatico (Figuras 5-6). La inmunohistoquímica fue positiva para sinaptofisina, cromogranina y CEA (Figura 7). La biopsia de los nódulos de la serosa gástrica fue compatible con un tumor del estroma gastrointestinal de $1 \times 1 \times 0,5 \mathrm{~cm}$, con reacción positiva para CD 117 (Figura 8) y negativa para proteína S-100 y actina.

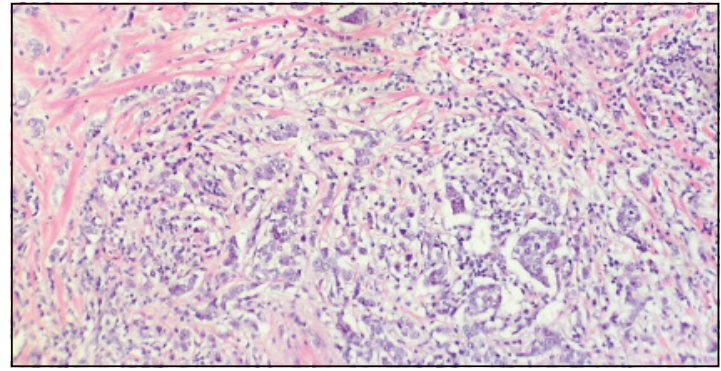

Figura 6. Microscopía de tumor duodenal con tinción de Hematoxilina- Eosina. 400X.
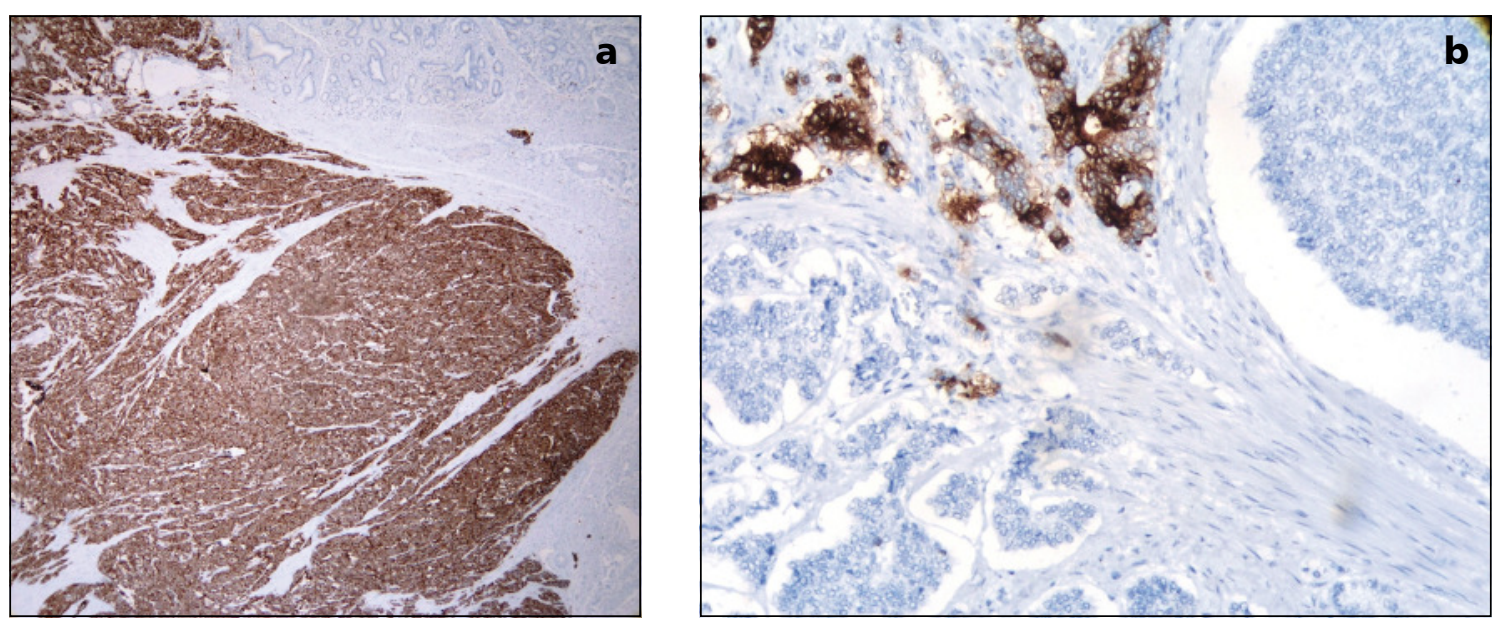

Figura 7. Evaluación de inmunohistoquímica de tumor duodenal, con marcación positiva para sinaptofisina 40X (a) y antígeno carcinoembrionario $400 \times(b)$ 


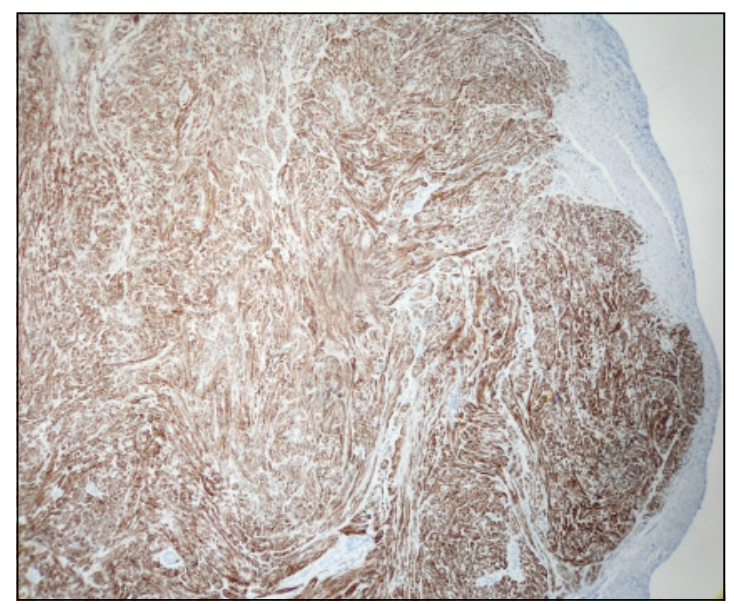

Figura 8. Tumor gástrico fusocelular con inmunohistoquímica positiva para CD 117, compatible con tumor del estroma gastrointestinal (GIST). 40x.

Se discutió el caso en comité de oncología y se decidió observación y control seriado con imágenes por tumores GIST de estómago. Actualmente la paciente se encuentra en buenas condiciones sin recidiva a los 4 meses de la cirugía.

\section{Discusión}

Los tumores gastrointestinales, son uno de los tipos de tumores a los que se asocia la NF1. Su real incidencia es difícil de evaluar, pero hay trabajos que indican que estarían presentes en un 10 a $25 \%$ de los pacientes con NF1. Se han descrito 3 tipos de tumores gastrointestinales en pacientes con $\mathrm{NF1}$; tumores del estroma gastrointestinal (GIST), tumores periampulares y neurofibromas. Los GIST son los más frecuentes, se ubican en intestino delgado y en el estómago, con una incidencia de $7 \%{ }^{4}$, lo que representa un riesgo de 150 veces mayor que en la población general. Los tumores periampulares son menos frecuentes que los GIST, pero se ha descrito un aumento en su incidencia ${ }^{5}$. Se estima que sólo $5 \%$ de estos tumores producen síntomas $^{6}$, y se manifiestan como sangrado gastrointestinal, dolor abdominal, obstrucción intestinal o ictericia obstructiva. En nuestro caso la paciente presentaba tanto múltiples tumores GIST del estómago como una neoplasia periampular, que fue la que dio síntomas.

Los casos de tumores periampulares en NF1 son raros. Se ha descrito, según una revisión de 37 pacientes, que la ubicación más frecuente es la ampolla, en $54 \%$, en segundo lugar duodenal, en $38 \%$ y $5 \%$ para cabeza de páncreas. Histológicamente, la mayoría de los tumores fueron carcinoides $(41 \%)$, seguidos por neurofibromas (30\%), neurofibrosarcomas (8\%), adenocarcinomas (8\%), schwanoma $5 \%$, paraganglioma $5 \%$ y ganglioneuroma con $3 \%{ }^{7}$. En el caso de nuestra paciente presentó una histología poco frecuente, un tumor mixto; adenocarcinoma y tumor neuroendocrino. En pacientes con NF1 esta histología es rara.

Existen varias alternativas descritas para el tratamiento de los tumores periampulares en pacientes con NF1. En casos de tumores neuroendocrinos menores a $2 \mathrm{~cm}$, superficiales, que tienen una baja probabilidad de tener linfonodos positivos podrían ser manejados con resección local, ya sea por vía endoscópica como quirúrgica. Esto es controversial y algunos autores recomiendan que esta alternativa sea reservada solo para pacientes añosos, con comorbilidades importantes y para tumores neuroendocrinos de alta diferenciación. Para pacientes con tumores mayores de $2 \mathrm{~cm}$, con buen riesgo quirúrgico el tratamiento es la pancreatoduodenectomía, tal como se optó en el caso que presentamos ${ }^{2,8}$. Por los escasos casos reportados en pacientes con NF1, no existen estudios que comparen las distintas alternativas y la decisión debe ser tomada caso a caso.

La sobrevida de los pacientes con NF1 con tumores periampulares resecados es elevada. Se describe una sobrevida del $90 \%$ a 5 años 9.

Se estima que un $1,5 \%$ de los tumores GIST ocurren en pacientes con $\mathrm{NF}^{2,10,11}$. Su diagnóstico es difícil por su localización submucosa. Se cree que son originados de las células de Cajal. Sus características moleculares están bien definidas, como c-Kit y PDGFR alfa (platelet derived growth factor receptor alpha) aunque en pacientes con $\mathrm{NF}$, las mutaciones típicas no aparecen, lo que sugiere una patogenia diferente. Pueden desarrollar anemia y sangramiento gastrointestinal y la mayoría de los tumores asociados a NF1, tiene un buen pronostico ${ }^{10}$.

El tratamiento de los tumores tipo GIST, es la resección quirúrgica completa con márgenes negativos. No requieren linfadenectomia porque la metástasis a linfonodos es muy rara ${ }^{9}$. La resección vía laparoscópica ha sido recomendada para tumores GIST gástricos. En una serie de 28 paciente con 
NF1 y tumores GIST, se demuestra que el pronóstico de los casos metastásicos es malo, y que la tasa de respuesta en este caso a imatinib es baja, por lo que pacientes con NF 1 y tumores GIST de riesgo intermedio-alto, no deberían ser elegidos para adyuvancia ni neoadyuvancia con imatinib ${ }^{12}$. En el caso que presentamos se optó por solo observación de los GIST gástricos, debido a que estos tumores eran asintomáticos, todos eran menores a $1 \mathrm{~cm}$ y para lograr una resección completa la paciente habría necesitado una gastrectomía total además de la pancreatoduodenectomia.

\section{Conclusión}

Los pacientes con NF1 presentan un riesgo elevado de desarrollar tumores periampulares y GIST del tubo digestivo. La mayor frecuencia de tumores periampulares en estos pacientes se originan en la ampolla y el duodeno. Los síntomas gastrointestinales y la baja de peso deben alertar sobre la posibilidad de aparición este tipo de tumores. Su tratamiento es controversial y la cirugía resectiva estaría indicada en la mayoría de los pacientes. La sobrevida es alta y los pacientes deben mantenerse en control por la posibilidad de aparición de otras neoplasias.

\section{Referencias}

1. Bajor J. Gastrointestinal stromal tumors in neurofibromatosis type 1. Orv Hetil 2009; 150: 149-53.

2. Relles D, Back J, Witkiewicz A, Yeo C. Periampullary and duodenal neoplasms in Neurofibromatosis Type 1: Two cases and an Updated 20 year Review of the literatura yielding 76 cases. J Gastrointest Surg 2010; 14:
1052-61.

3. Pinson S, Wolkenstein P. Neurofibromatosis type 1 or Von Recklinghausen's disease. Rev Med Interne 2005; 26: 196-215.

4. Zöller ME, Rembeck B, Odén A, Samuelsson M, Angervall L. Malignant and benign tumors in patients with neurofibromatosis type 1 in a defined Swedish population. Cancer 1997; $79: 2125-31$.

5. Bettini R, Falconi M, Crippa S, Capelli P, Boninsegna L, Pederzoli P. Ampullary somatostatinoma and jejunal gastrointestinal stromal tumor in a patient with Von Recklinghausen' disease. World J Gastroenterol 2007; $13: 2761-3$.

6. Pinsk I, Dukhno O, Ovnat A, Levy I. Gastrointestinal complications of von Recklinghausen's disease: two case reports and a review of the literature. Scand J Gastroenterol 2003; 38: 1275-8.

7. Klein A, Clemens J, Cameron J. Periampullary neoplasms in von Recklinghausen's disease. Surgery 1989; 106: 815-9.

8. Hartel M, Wente MN, Sido B, Friess H, Büchler MW. Carcinoid of the ampulla of Vater. J Gastroenterol Hepatol 2005; 20: 676-81.

9. Buck L, Perry B, Richards M. Periampullary Carcinoid Tumor in a Woman with neurofibromatosis. Curr Surg 2006 ; 63: 252-4.

10. Ferner R. Neurofibromatosis 1 and neurofibromatosis 2 : a twenty first century perspective review. Lancet Neurol 2007; 6: 340-51.

11. Miettinen M, Fetsch JF, Sobin LH, Lasota J. Gastrointestinal stromal tumors in patients with neurofibromatosis 1: a clinicopathologic and molecular genetic study of 45 cases. Am J Surg Pathol 2006 ; 30: 90-6.

12. Mussi C, Schildhaus H, Gronchi A, Wardelmann E, Hohenberger P. Therapeutic consequences from molecular biology for gastrointestinal stromal tumor patients affected by neurofibromatosis type 1 . Clin Cancer Res 2008; 14: 4550-5. 